\title{
Race-based perceptual asymmetries underlying face processing in infancy
}

\author{
Angela Hayden, Ramesh S. Bhatt, Nicole Zieber, and Ashley Kangas \\ University of Kentucky, Lexington, Kentucky
}

\begin{abstract}
Adults process other-race faces differently than own-race faces. For instance, a single other-race face in an array of own-race faces attracts Caucasians' attention, but a single own-race face among other-race faces does not. This perceptual asymmetry has been explained by the presence of an other-race feature in other-race faces and its absence in own-race faces; this difference is thought to underlie race-based differences in face processing. We examined the developmental origins of this mechanism in two groups of Caucasian 9-month-olds. Infants in the experimental group exhibited a preference for a pattern containing a single Asian face among seven Caucasian faces over a pattern containing a single Caucasian face among seven Asian faces. This preference was not driven by the majority of elements in the images, because a control group of infants failed to exhibit a preference between homogeneous patterns containing eight Caucasian versus eight Asian faces. The results demonstrate that an other-race face among own-race faces attracts infants' attention but not vice versa. This perceptual asymmetry suggests that the other-race feature is available to Caucasians by 9 months of age, thereby indicating that mechanisms of specialization in face processing originate early in life.
\end{abstract}

The other-race effect (ORE) is the tendency of racial information to disrupt the processing of faces of other races. In effect, adults tend to distinguish between ownrace faces (i.e., faces in their own racial category) more easily than between other-race faces (i.e., faces that are not in their own racial category; see, e.g., Meissner \& Brigham, 2001; Rhodes, Hayward, \& Winkler, 2006; Walker \& Tanaka, 2003).

Although a number of models have been proposed to account for the ORE (see Meissner \& Brigham, 2001, for a review), Levin's (1996, 2000) model is particularly germane to the present research. Adults, according to Levin, process other-race faces less than own-race faces presumably because the former faces are from a less socially relevant out-group, whereas the latter are from a more socially relevant in-group (see also Corenblum \& Meissner, 2006). Levin argued that the adult perceptual system accomplishes this kind of differential processing by treating other-race information like a fundamental feature. Own-race information lacks this particular feature, and therefore, one's own race serves as the default category. This relationship between other-race and own-race faces is much like that between an oblique line and a vertical line: The tilt of an oblique line is considered a feature, whereas the absence of a tilt in the vertical line represents the lack of a feature.

Levin's $(1996,2000)$ studies showing that race is a fundamental feature for Caucasian adults were based on the Treisman and Gormican (1988) findings in visual search tasks that feature-positive targets (i.e., targets containing fundamental features, such as oblique lines) are more rapidly identified among feature-negative distractors (i.e., distractors lacking features, such as vertical lines) than vice versa. In Levin's studies, Caucasian participants exhibited an asymmetry in identifying the presence of a target face when they were asked to identify a single African American target face (a feature-positive target) among several Caucasian faces (feature-negative distractors) versus a single Caucasian face (feature-negative target) among several African American faces (feature-positive distractors). That is, these participants identified the presence of a unique face much more rapidly when it was African American than when it was Caucasian. Thus, Levin argued, the feature of race among own-race faces was more easily identified than vice versa by Caucasians.

The intent of the present study was to examine the developmental origins of the mechanism described above. To date, three studies have investigated the presence of the ORE in infancy. Sangrigoli and de Schonen (2004) habituated 3-month-old Caucasian infants to a female face, either Caucasian or Asian, and tested them with the familiar face paired with a novel face of the same race. The infants discriminated in the Caucasian condition but failed to discriminate in the Asian condition. Using Caucasian/Asian morphed stimuli, Hayden, Bhatt, Joseph, and Tanaka (2007) obtained data consistent with the Sangrigoli and de Schonen conclusion that 3-montholds exhibit the ORE. A study by Kelly et al. (2007) suggested that the ORE may not be robust until 9 months of age. Caucasian 3-month-olds failed to exhibit an ORE in

R.S.Bhatt, rbhatt@email.uky.edu 
this study, whereas 6-month-olds exhibited an ORE with Middle Eastern and African faces, but not with Asian faces. Nine-month-olds, however, exhibited the ORE with Asian faces as well, suggesting that the ORE is quite well developed at 9 months of age. This apparent strengthening of the ORE over time in infancy begs the question of how other-race faces are perceived by infants. As discussed above, the research conducted by Levin $(1996,2000)$ and by MacLin and Malpass (2001) suggested that the key difference in the processing of other-race versus own-race faces is the rapid segregation of the former on the basis of the other-race feature. One question that arises is whether a similar mechanism underlies the ORE in infancy also. We addressed this issue in the present study. It capitalized on the finding that infant attention is drawn to featural discrepancies in visual images.

Research with both adults and infants (e.g., Bhatt, 1997; Quinn \& Bhatt, 1998; Rovee-Collier, Hankins, \& Bhatt, 1992; Treisman \& Gormican, 1988) has suggested that discrepancies based on fundamental features attract attention. For example, when 3- to 4-month-olds in Quinn and Bhatt were familiarized to a display of $25+\mathrm{s}$, they subsequently exhibited a preference for a display containing a single feature discrepancy (an L among $24+$ s) over another display containing a single familiar + surrounded by 24 novel Ls. In other words, infants' performance was determined by a single pop-out element, rather than by the large number of surrounding elements in the array, thereby indicating attentional engagement by the discrepant element. Subsequent research by Bhatt, Hayden, Reed, Bertin, and Joseph (2006) showed that infants also exhibit asymmetries in discrepancy detection. In that study, infants detected concave elements among convex distractors, but not vice versa. This is analogous to the finding that, for adults, concavities (signals for object parts) pop out of displays when surrounded by convex elements but not vice versa (Hulleman, te Winkel, \& Boselie, 2000). Thus, prior studies have demonstrated not only that infants' attention is drawn to discrepancies, but also that, in infancy, as in adulthood, attention can be asymmetrical, so that feature-positive elements amid featurenegative elements attract attention but feature-negative elements amid feature-positive elements do not.

As discussed earlier, Levin's (1996) studies indicated asymmetries in Caucasians' detection of faces of different races, analogous to the asymmetries exhibited by infants. In the present study, we examined whether a similar racebased asymmetry would be exhibited by 9-month-olds. We chose to use 9-month-olds because Kelly et al. (2007) suggested that the ORE may not be robust until this age. Infants in the experimental condition were exposed to a pattern containing a single Asian face among seven Caucasian faces paired with another pattern containing a single Caucasian face among seven Asian faces (Figure 1). Given that discrepancies attract infants' attention (as was discussed above), if an Asian face amid Caucasian faces attracts attention but a Caucasian face among Asian faces does not, infants should look longer at the former image than at the latter image. It is also possible, of course, that infants could look longer at the Asian-among-Caucasian display than at the Caucasian-among-Asian display be- cause of the seven Caucasian faces in the former display versus the seven Asian faces in the latter display. In other words, performance might be driven by the majority of faces in the displays, rather than by the singleton discrepant faces. To examine this possibility, a control group of infants was tested for preference between a pattern containing all Caucasian faces and a pattern containing all Asian faces (Figure 1). If the infants in the experimental condition exhibited a preference for the single Asian-amongCaucasian pattern, and if this preference was significantly different from the preference for the homogeneous Caucasian pattern in the control condition, then it would be strong evidence that Asian faces among Caucasian faces did attract the infants' attention to a greater degree than did the Caucasian-among-Asian pattern. Such a result would indicate that, in infancy, as in adulthood, other-race faces are feature-positive stimuli that attract attention when surrounded by own-race faces, whereas own-race faces are feature-negative stimuli that do not attract attention when surrounded by other-race faces.

\section{METHOD}

\section{Participants}

Thirty-two Caucasian 9-month-old participants (mean age $=$ 271.69 days, $S D=7.82 ; 12$ female) were recruited through birth announcements, by word of mouth, and from a local hospital. Data from 2 additional infants were excluded, 1 due to position preference ( $95 \%$ or more looking to one side), and another for sibling interference.

\section{Stimuli}

The stimuli were color photographs of four female Asian and four female Caucasian faces exhibiting neutral emotions (see Figure 1). Two of the Asian faces and two of the Caucasian faces were from the JACNeuf set (Matsumoto \& Ekman, 1988). The Asian faces were N48 and N43 from this set; the Caucasian faces were N25 and N16. The other four faces (two Asian, two Caucasian) were taken from the MacBrain set. (Development of the MacBrain set was overseen by Nim Tottenham and supported by the John D. and Catherine T. MacArthur Foundation Research Network on Early Experience and Brain Development. Please contact Nim Tottenham at tott0006@tc.umn .edu for more information.) We are unable to publish photographs from the MacBrain set because we could not obtain permission to do so. However, the Asian faces are listed as Faces 16 and 19 and the Caucasian faces are listed as Faces 2 and 8 on the MacBrain Web site. An individual Asian face was paired with an individual Caucasian face from the same face bank to form a total of four pairs. The same face pairs were used in the experimental and control conditions. Using Adobe Photoshop, the hair was removed from the head (this is a common practice in studies investigating the ORE [e.g., Hayden et al., 2007; Walker \& Tanaka, 2003]). In addition, in order to avoid pop-out based on low-level features, we equated for skin tone within the pairs of faces. In two of the Asian-Caucasian face pairings, the skin tones of the Asian faces were matched to those of the corresponding Caucasian faces; in the other two pairings, the skin tones of the Caucasian faces were matched to those of the Asian faces. Thus, equal numbers of Asian and Caucasian faces had natural and artificial skin tones, but within each Asian-Caucasian pair, the skin tones matched.

Eight faces were arranged on a black background in a diamond formation (as in Levin's [1996, 2000] studies; see Figure 1). Faces were located $0.76^{\circ}$ apart from one another. The diamond-shaped face pattern subtended approximately $22^{\circ} \times 17.25^{\circ}$. Individual faces subtended approximately $3.69^{\circ} \times 5.60^{\circ}$. Homogeneous displays were created for the control condition, and pop-out displays for the ex- 


\section{Experimental Condition}

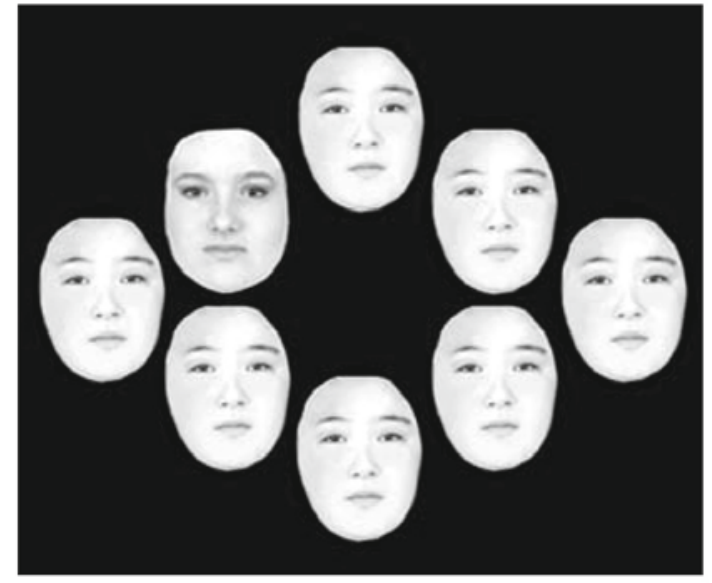

Caucasian-Among-Asian Display

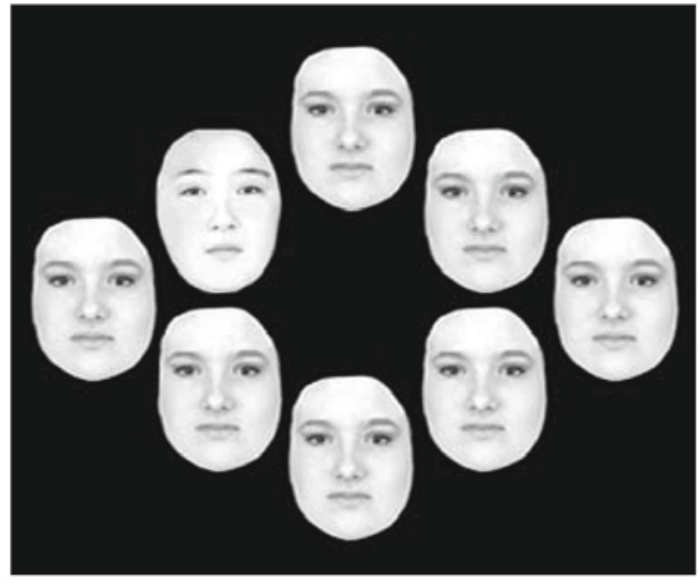

Asian-Among-Caucasian Display

\section{Control Condition}

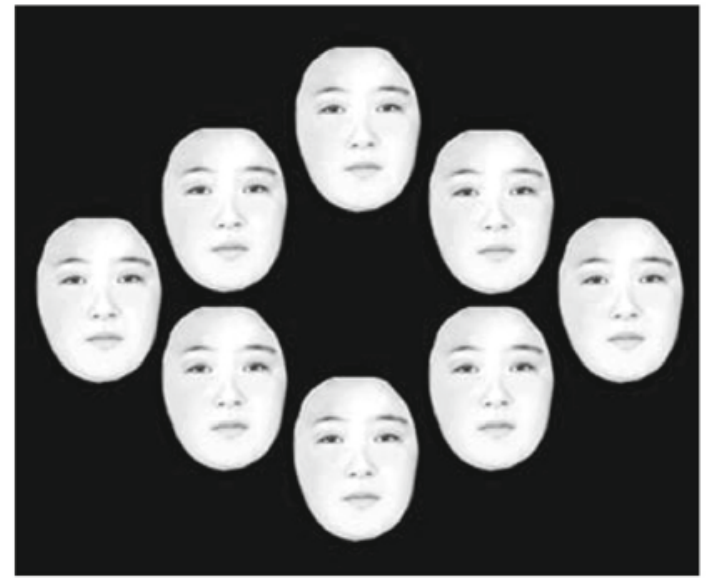

Asian Face Display

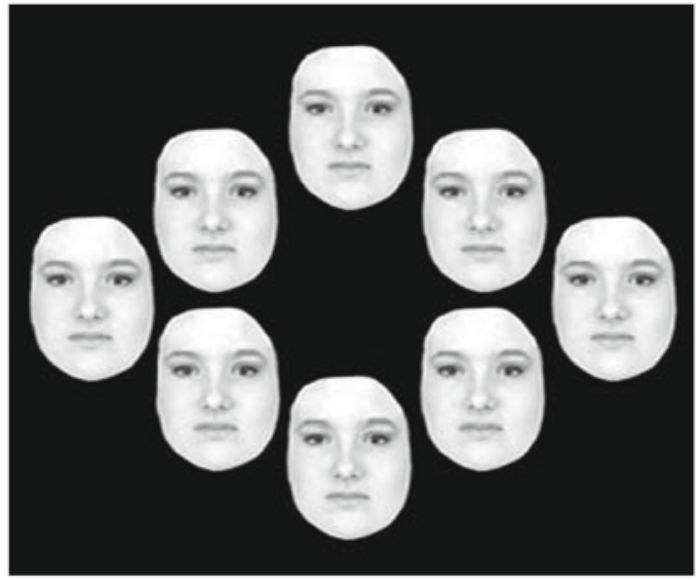

Caucasian Face Display

Figure 1. Examples of the face pairs used in the present experiment. In the experimental condition, infants saw an Asian face among Caucasian faces paired with a Caucasian face among Asian faces. In the control condition, infants saw a homogeneous display of Asian faces paired with a homogeneous display of Caucasian faces. Preference for the majority Caucasian image was calculated for each condition (i.e., the Asian-among-Caucasian display in the experimental condition and the homogeneous Caucasian face display in the control condition). In two of the face pairs, the skin tones of the Asian faces were changed to match those of the corresponding Caucasian faces (as in the example shown in this figure); in two others, the skin tones of the Caucasian faces were changed to match Asian faces. The actual face patterns shown to the infants were in color.

perimental condition. Homogeneous displays assessed overall preference for groups of Caucasian over Asian faces: They contained eight identical Asian or Caucasian faces (Figure 1). In the experimental condition, the Caucasian-among-Asian displays consisted of a single Caucasian face among seven Asian distractors, whereas the Asian-among-Caucasian displays consisted of a single Asian face among seven Caucasian faces (Figure 1). The singleton Caucasian and Asian faces in these patterns were in the same location within face pairings but varied between face pairings.

\section{Procedure}

The infants were tested using a spontaneous preference procedure that has commonly been employed in infancy studies (e.g., Kelly et al., 2005). They were randomly assigned to the experimental and control conditions. In addition, within each group, the infants were randomly assigned to one of the four Asian-Caucasian face pairings.

The infants were seated in a darkened chamber, approximately $45 \mathrm{~cm}$ in front of a 20 -in. computer monitor. They were tested on a total of two spontaneous preference trials, each of which lasted $8 \mathrm{sec}$. Each trial began with the presentation of an attention-getter (rapidly alternating shapes) in the center of the screen. When the infant's attention was drawn to the center of the screen, the shapes disappeared, and a paired display of faces appeared on the screen. The left-right locations of the Caucasian/Asian and the Caucasian-among-Asian/ Asian-among-Caucasian patterns were randomly determined and counterbalanced across the set of infants in each condition; this location was changed from one trial to the next, to avoid side bias. 
A video camera, located on top of the computer monitor, and an associated DVD recorder were used to record the infants' behavior. Coding of the infants' performance was conducted offline, with the coder unaware of the left-right location of the stimulus patterns. The DVD player was slowed to $20 \%$ of the normal speed during coding. A separate coder recoded data from 6 infants in order to obtain a reliability measure. The Pearson correlation between the two observers' scores was .92 .

\section{RESULTS}

Preference during the test trials was assessed by computing a percentage score that measured preference for the display in which the majority of the pattern consisted of Caucasian faces (i.e., the homogeneous Caucasian display in the control condition, and the Asian-among-Caucasian display in the experimental condition). This score was computed by dividing the total duration of looking time for the Caucasian majority display by the total duration of looking time for both displays and multiplying this ratio by 100 to obtain a percentage. In effect, these scores indicated how much the infants in the experimental group preferred the Asian-among-Caucasian display over the Caucasian-among-Asian display and how much the infants in the control group preferred the homogeneous Caucasian display over the homogeneous Asian display.

See Table 1 for preference scores. Note that the score in the experimental condition was greater than that in the control condition. In order to explore group differences, as well as to ensure that individual face pairs did not differ from one another, we conducted a face pair (Face Pair 1, Face Pair 2, Face Pair 3, Face Pair 4) $\times$ group (experimental, control) ANOVA. The results indicated a significant group main effect, indicating that infant preference was significantly different between the control and experimental groups $\left[F(1,30)=8.88, p<.01, \eta_{\mathrm{p}}^{2}=.27\right]$. Neither the face pair main effect nor the face pair $\times$ group interaction effect was significant (both $F \mathrm{~s}<2.00, p \mathrm{~s}>.15$ ), indicating that the infants performed similarly in their assigned groups, irrespective of face pair. Recall that two of the four faces of each race were tested with the natural skin tone, whereas the others had their skin tone changed to match their corresponding opposite race faces. The fact that face pair was not a statistically significant main or interaction factor therefore indicates that the preference for the Asian-among-Caucasian pattern over the reverse pattern exhibited in the experimental condition and the lack of preference between the homogeneous patterns exhibited in the control condition (see below) did not vary as a function of whether the faces of the two races had natural or unnatural skin tones. In other words, performance was driven by the physiognomic characteristics of faces, rather than by any "weirdness" factor based on the mismatch between races and their skin tone.

Single-sample $t$ tests comparing the control and experimental groups' means against the chance level of $50 \%$ indicated that the infants in the experimental group preferred the Asian-among-Caucasian patterns to the Caucasianamong-Asian patterns significantly above chance level $[t(15)=2.73, p<.02$, two-tailed], but the infants in the control group did not significantly prefer either of the homogeneous displays $[t(15)=-1.40, p>.15$, two-tailed]. The fact that the infants in the control condition failed to exhibit a preference between the homogeneous groups of Caucasian versus Asian faces, combined with the fact that the infants in the experimental condition preferred the Asian-among-Caucasian pattern over the Caucasianamong-Asian pattern, suggests that the single Asian face among Caucasian faces attracted the infants' attention more than did the single Caucasian face among Asian faces. Thus, for Caucasian infants, as for adults, otherrace faces appear to have a distinctive other-race feature that captures attention, whereas own-race faces do not seem to have the same attention-capturing feature.

Table 1

Mean Look Durations for Each Face Pattern and Overall Preference Scores for the Majority Caucasian Display in the Experimental and Control Conditions

\begin{tabular}{|c|c|c|c|c|c|}
\hline & \multicolumn{2}{|c|}{ Look Durations (sec) } & \multicolumn{3}{|c|}{$\begin{array}{c}\text { Preference for } \\
\text { Majority Caucasian Display } \\
\end{array}$} \\
\hline & \multirow{2}{*}{$\begin{array}{l}\text { Caucasian } \\
\text { Majority }\end{array}$} & \multirow{2}{*}{$\begin{array}{l}\text { Asian } \\
\text { Majority }\end{array}$} & \multicolumn{2}{|c|}{ Percentage } & \multirow[b]{2}{*}{$t$} \\
\hline & & & $M$ & $S D$ & \\
\hline \multicolumn{6}{|l|}{ Asian skin tone on both faces } \\
\hline \multicolumn{6}{|l|}{ MacBrain Faces 8, 19} \\
\hline Experimental condition & 30.36 & 23.47 & 56.62 & 5.29 & $\mathrm{n} / \mathrm{a}$ \\
\hline Control condition & 27.92 & 28.41 & 49.38 & 10.33 & $\mathrm{n} / \mathrm{a}$ \\
\hline \multicolumn{6}{|l|}{ MacBrain Faces 2, 16} \\
\hline Experimental condition & 28.08 & 29.12 & 49.14 & 9.75 & $\mathrm{n} / \mathrm{a}$ \\
\hline Control condition & 25.95 & 34.06 & 43.13 & 7.96 & $\mathrm{n} / \mathrm{a}$ \\
\hline \multicolumn{6}{|c|}{ Caucasian skin tone on both faces } \\
\hline \multicolumn{6}{|c|}{ JACNeuf Faces N16, N43 } \\
\hline Experimental condition & 32.94 & 23.72 & 58.11 & 10.37 & $\mathrm{n} / \mathrm{a}$ \\
\hline Control condition & 27.54 & 28.68 & 48.99 & 4.13 & $\mathrm{n} / \mathrm{a}$ \\
\hline \multicolumn{6}{|l|}{ JACNeuf Faces N25, N48 } \\
\hline Experimental condition & 32.17 & 23.70 & 57.54 & 2.60 & $\mathrm{n} / \mathrm{a}$ \\
\hline Control condition & 27.13 & 28.15 & 49.00 & 2.38 & $\mathrm{n} / \mathrm{a}$ \\
\hline \multicolumn{6}{|l|}{ Overall Preference } \\
\hline Experimental condition & 30.89 & 25.00 & 55.35 & 7.85 & $2.73^{*}$ \\
\hline Control condition & 27.13 & 29.82 & 47.62 & 6.77 & -1.40 \\
\hline
\end{tabular}




\section{DISCUSSION}

The results of the present study demonstrate that an other-race face among own-race faces attracts Caucasian infants' attention but not vice versa. This asymmetry in attentional engagement is consistent with Levin's (1996) findings that Caucasian adults detect an African American face among Caucasian faces more readily than a Caucasian face among African American faces. Levin explained his findings by suggesting that, for adults, other-race faces contain a fundamental other-race feature that is lacking in own-race faces and this leads to the faster detection of an other-race face among own-race faces than vice versa, because feature-positive elements are detected more easily among feature-negative elements than the reverse. The present findings thus suggest that the developmental origin of this asymmetry in the classification of other-race versus own-race faces is evident as early as 9 months of age in Caucasian infants: Even at this age, other-race faces appear to have an other-race feature that is not available in own-race faces.

We hasten to add that our findings, although consistent with Levin's $(1996,2000)$ findings, are not exactly the same, because we did not measure the infants' speed of processing. However, our findings are strongly indicative of featural asymmetry in other-race versus own-race faces, because they not only are consistent with Levin's findings, but also are consistent with previous research indicating that featural discrepancies attract infants' attention (e.g., Bhatt, 1997) and that a feature-positive element among feature-negative elements attracts infants' attention but not vice versa (Bhatt et al., 2006).

One limitation of the present study is the fact that only Caucasian infants were tested. The use of infants of at least two races (in the present case, Asian in addition to Caucasian) would allow the demonstration of asymmetrical pop-out effects in both directions and, thus, rule out the possibility that some low-level nonracial feature associated with the Asian faces and not the Caucasian faces used in the present study led to the perceptual asymmetry exhibited in this study. We also wish to note, however, that the infants in the control condition, who were tested with homogeneous arrays of faces, failed to exhibit a preference. Thus, any low-level nonracial feature that may have led to the present pattern of performance must be akin to the other-race feature in attracting attention when embedded in an array of discrepant elements, rather than when presented in homogeneous arrays. The probability of the presence of such a nonracial fundamental feature in the Asian faces is likely not very high, although the present research cannot rule out such a possibility. Moreover, two of the face pairs used in the present study (those from the JACNeuf set) are part of a stimulus set in which adults exhibited crosscultural performance differences based on race (Beihl et al., 1997). These factors provide some confidence that the infants' performance in the present study was based on racial features.

Prior research has shown that, like Caucasians, adult African Americans detect African American faces among
Caucasian faces more quickly than vice versa (Chiao, Heck, Nakayama, \& Ambady, 2006). In other words, visual search by African Americans shows the same asymmetry as that exhibited by Caucasian participants rather than the opposite, which would be predicted by a simple own-race/other-race feature model. These findings have been explained by suggesting that factors besides race (such as the minority/majority nature of groups and the need for individuation) might affect the direction of or preclude perceptual asymmetries (Levin, 1996). Thus, although the present research suggests that 9-month-old Caucasian infants exhibit a perceptual asymmetry that is similar to the asymmetry exhibited by Caucasian adults, this kind of asymmetry may not be exhibited by infants of other races and from different cultural contexts.

Another point to note concerns the fact that infants in prior studies by Bar-Haim, Ziv, Lamy, and Hodes (2006) and by Kelly et al. (2005) exhibited a preference for own-race faces but infants in the control condition of the present study failed to exhibit a preference between homogeneous arrays of own-race and other-race faces. The present study involved 9-month-olds, whereas prior studies tested 3-month-olds. Also, the present study contrasted multiple sets of faces, whereas prior studies involved preference tests between individual faces. Moreover, skin tones were matched between pairs of Caucasian and Asian faces in the present study, but not in previous studies. In addition, the stimuli used in the present study did not include external features (hair, ears), whereas those used by Bar-Haim et al. and Kelly et al. did. Any one or a combination of these factors may account for the differences between the present results and those of Bar-Haim et al. and Kelly et al.

In conclusion, the present study indicates that otherrace information is a feature that draws attention by 9 months of age in Caucasian infants. Although this result does not necessarily imply that other-race faces are rapidly designated as part of an out-group and, thus, processed shallowly, as is theorized to be the case in adult Caucasian other-race processing (Levin, 1996, 2000; MacLin \& Malpass, 2001), it does indicate that other-race faces are categorized and processed differently from ownrace faces even in infancy in Caucasians. The fact that just 9 months of experience is enough for Caucasian infants to develop an other-race feature is consistent with other reports of perceptual narrowing and specialization during the first year of life - specifically, poorer discrimination of other-race (Kelly et al., 2007) and other-species (Pascalis, de Haan, \& Nelson, 2002) faces by older than by younger infants. Future studies should examine the nature of the cognitive economy that results from such rapid specialization early in life (Kuhl, Conboy, Padden, Nelson, \& Pruitt, 2005).

\section{AUTHOR NOTE}

This research was supported by Grant HD042451 from the National Institute of Child Health and Human Development. We thank the infants and parents who participated in this study. Address correspondence to R. S. Bhatt, Psychology Department, University of Kentucky, Lexington, KY 40506-0044 (e-mail: rbhatt@email.uky.edu). 


\section{REFERENCES}

Bar-Haim, Y., Ziv, T., Lamy, D., \& Hodes, R. M. (2006). Nature and nurture in own-race face processing. Psychological Science, 17, 159-163.

Beinl, M., Matsumoto, D., Ekman, P., Hearn, V., Heider, K., KudoH, T., \& Ton, V. (1997). Matsumoto and Ekman's Japanese and Caucasian facial expressions of emotion (JACFEE): Reliability data and cross-national differences. Journal of Nonverbal Behavior, 21, 3-21.

Bнатт, R. S. (1997). The interface between perception and cognition: Feature detection, visual pop-out effects, feature integration, and longterm memory in infancy. Advances in Infancy Research, 11, 143-191.

Bhatt, R. S., Hayden, A., Reed, A., Bertin, E., \& Joseph, J. E. (2006). Infants' perception of information along object boundaries: Concavities versus convexities. Journal of Experimental Child Psychology, 94, 91-113.

Chiao, J. Y., Heck, H. E., Nakayama, K., \& Ambady, N. (2006). Priming race in biracial observers affects visual search for black and white faces. Psychological Science, 17, 387-392.

Corenblum, B., \& Meissner, C. A. (2006). Recognition of ingroup and outgroup children and adults. Journal of Experimental Child Psychology, 93, 187-206.

Hayden, A., Bhatt, R. S., Joseph, J. E., \& Tanaka, J. W. (2007). The other-race effect in infancy: Evidence using a morphing technique. Infancy, 12, 95-104.

Hulleman, J., te Winkel, W., \& Boselie, F. (2000). Concavities as basic features in visual search: Evidence from search asymmetries. Perception \& Psychophysics, 62, 162-174.

Kelly, D. J., Quinn, P. C., Slater, A. M., Lee, K., Ge, L., \& Pascalis, O. (2007). The other-race effect develops during infancy: Evidence of perceptual narrowing. Psychological Science, 18, 1084-1089.

Kelly, D. J., Quinn, P. C., Slater, A. M., Lee, K., Gibson, A., SMith, M., ET AL. (2005). Three-month-olds, but not newborns, prefer own-race faces. Developmental Science, 8, F31-F36.

Kuhl, P. K., Conboy, B. T., Padden, D., Nelson, T., \& Pruitt, J. (2005). Early speech perception and later language development: Implications for the "critical period." Language Learning \& Development, 1, 237-264.

LEvin, D. T. (1996). Clarifying faces by race: The structure of face cat- egories. Journal of Experimental Psychology: Learning, Memory, \& Cognition, 22, 1364-1382.

LEvin, D. T. (2000). Race as a visual feature: Using visual search and perceptual discrimination tasks to understand face categories and the cross-race recognition deficit. Journal of Experimental Psychology: General, 129, 559-574.

MacLin, O. H., \& MaLPass, R. S. (2001). Racial categorization of faces: The ambiguous race face effect. Psychology, Public Policy, \& Law, 7, 98-118.

Matsumoto, D., \& Ekman, P. (1988). Japanese and Caucasian facial expressions of emotion (JACFEE) [Slides]. San Francisco: San Francisco State University, Department of Psychology, Intercultural and Emotion Research Laboratory.

Meissner, C. A., \& Brigham, J. C. (2001). Thirty years of investigating own-race bias in memory for faces: A meta-analytic review. Psychology, Public Policy, \& Law, 7, 3-35.

Pascalis, O., De HaAn, M., \& Nelson, C. A. (2002). Is face processing species-specific during the first year of life? Science, 296, 13211323.

QuinN, P. C., \& BhatT, R. S. (1998). Visual pop-out in infants: Convergent evidence and an extension. Infant Behavior \& Development, 21, 273-288.

RHODES, G., HAYWARD, W. G., \& WinKLER, C. (2006). Expert face coding: Configural and componential coding of own-race and other-race faces. Psychonomic Bulletin \& Review, 13, 499-505.

Rovee-Collier, C., Hankins, E., \& Bhatt, R. S. (1992). Textons, visual pop-out effects, and object recognition in infancy. Journal of Experimental Psychology: General, 121, 435-445.

SANGRIGOLI, S., \& DE SCHONEN, S. (2004). Recognition of own-race and other-race faces by three-month-old infants. Journal of Child Psychology \& Psychiatry, 45, 1219-1227.

TrEISMAN, A., \& GormicAN, S. (1988). Feature analysis in early vision: Evidence from search asymmetries. Psychological Review, 95, 15-48.

Walker, P. M., \& TANaKa, J. W. (2003). An encoding advantage for own-race versus other-race faces. Perception, 32, 1117-1125.

(Manuscript received August 1, 2008; revision accepted for publication October 31, 2008.) 